Instituto Internacional de Investigación y Desarrollo Tecnológico Educativo INDTEC, C.A.

DOI: https://doi.org/10.29394/scientific.issn.2542-2987.2016.1.1.12.212-230

OAI-PMH: http://www.indteca.com/ojs/index.php/Revista Scientific/oai

\title{
Reutilización de Residuos Sólidos en la Promoción de la Cultura Ambiental
}

Autora: Isbely del Carmen Aguilera Osorio Universidad Pedagógica Experimental Libertador, UPEL Im19752005@gmail.com

Barinas, Venezuela

\section{Resumen}

El presente estudio tuvo como objetivo proponer un plan estratégico para la reutilización de los residuos sólidos en la promoción de la cultura ambientalista en los estudiantes del sexto grado de la Escuela Básica Estadal "Chaparral", ubicada en el municipio Pedraza del Estado Barinas para el año escolar 2015-2016. Investigación de naturaleza cuantitativa, de tipo descriptiva en la modalidad de proyecto factible, estructurado en cinco fases correspondientes al diagnóstico, la factibilidad, diseño, aplicación y evaluación de los resultados en la aplicación de un plan estratégico basado en la reutilización de residuos sólidos. Con relación a la población, esta se circunscribe a cinco docentes, seleccionando la totalidad para la muestra por ser un universo pequeño. Como técnica se empleó la encuesta, se aplicó un instrumento tipo cuestionario de 8 interrogantes en escala Likert de tres opciones de respuesta. Para la confiabilidad se aplicó la fórmula de Coeficiente (a) Alfa de Cronbach, la validez a través de juicio de expertos. Como conclusiones se configuró una propuesta denominada Plan estratégico para la reutilización de los residuos sólidos en la promoción de la cultura ambientalista en los estudiantes del sexto grado de la Escuela Básica Estadal "Chaparral", durante el año escolar 2015-2016.

Palabras clave: reutilización de residuos sólidos; cultura ambientalista; plan estratégico. 


\section{Reuse of Solid Waste in The Promotion of Culture Environment}

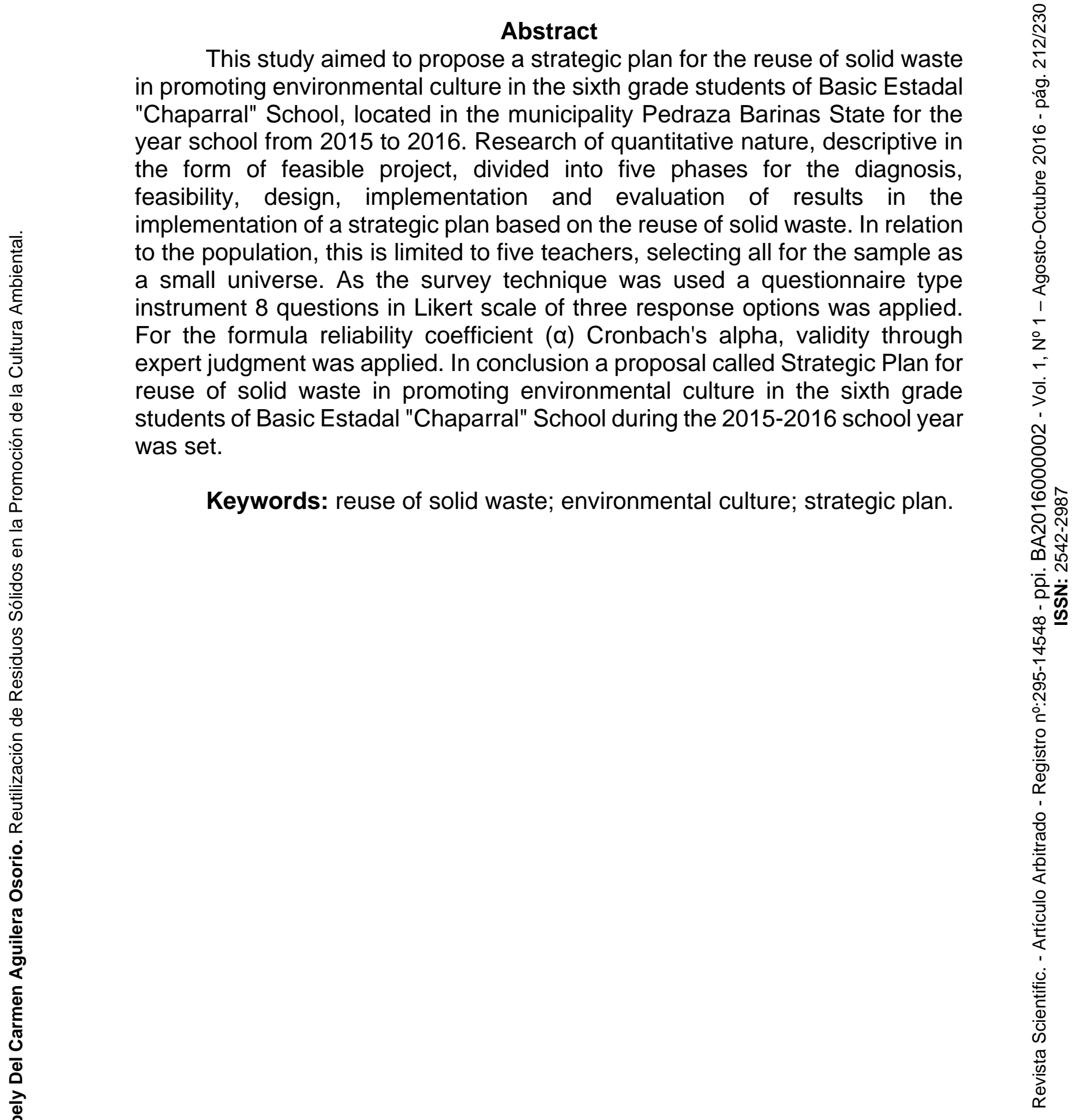

Date Received: 12-06-2016 Date Acceptance: 18-07-2016 


\section{A modo de Introducción}

El desarrollo de la sociedad humana, ha establecido un marcador determinado por la presencia de un alto grado de contaminación, que durante mucho tiempo el hombre ha causado, a través de sus prácticas diarias de tipo doméstico, comercial, industrial; requiere de procesos sencillos o complejos que generan una diversidad de productos e igualmente de desechos que consideran como inservibles, pero que tienen una gran utilidad; a estos se les denomina: residuos. Dentro de estos residuos encontramos diferentes tipos; clasificados de acuerdo a su estado (liquido, sólido, gaseoso), a su origen (residencial, comercial, industrial, entre otros.), a su manejo (peligrosos e inertes) y por último a su composición (orgánicos e inorgánicos, producto del crecimiento exponencial de la tecnología aplicada en la elaboración producción distribución y aprovechamiento de bienes y servicios. Por esta razón la reutilización de los residuos sólidos es de gran importancia para el manejo de una cultura ambientalista dentro de la sociedad.

En este entorno, adecuar los procesos de enseñanza y aprendizaje a estos cambios contantes y determinar un sistema de modelado que se inserte en las nuevas necesidades axiológicas se ha convertido en un problema constante para el sistema educativo y para la escuela en sí misma, que este trabajo pretende comprender y mejorar, en función de las condiciones y expectativas de la institución educativa.

Ahora bien, con el desarrollo de la actividad industrial y el crecimiento de la población, se dieron las condiciones para el incremento de los factores de desequilibrio ambiental que caracterizan a la época actual. Consumo masivo, escalada de la producción, mecanismos facilitadores de la distribución de elementos generadores de residuos, así como el requerimiento de nuevos productos, se traducen en un estado de diversos grados de contaminación o al menos de problemáticas asociadas al aprovechamiento efecto de los recursos naturales en el planeta. Esta es una realidad fundamental en el 
mundo actual, que ha derivado en el interés por un cambio paradigmático de la actividad humana en relación con el ambiente.

En este aspecto, Latinoamérica ha representado un espacio de doble interés, reflejado en la opinión de Valero (2011) quien afirma: "La Educación Ambiental es un interés funcional en la vida de la región, habida cuenta de la presencia de recursos naturales considerables y de la necesidad de generar conciencia en cuanto a su protección" (pág. 131) Para el autor, en esta zona del mundo se concentran las mayores reservas de recursos naturales del mismo, pero también, un requerimiento importante de formación ambiental dirigida a la protección de los mismos, a través de la emergencia de nuevos valores y conductas ambientalistas, enmarcadas en una cultura del aprovechamiento consciente de la naturaleza.

Cabe señalar, que en la región, países como Argentina, han desarrollado planes conjuntos de formación ambiental en espacios escolares, a través de proyectos como el planteado por Yélamos (2013) en el que señala: "La Secretaria de Ambiente y Desarrollo Sustentable de la Nación, define la necesidad de incorporar una carga de valores ambientales desde la propia escuela a través de mecanismos interactivos y estratégicos" (pág. 22). Al respecto plantea alternativas como la reutilización de residuos sólidos y el juego, con los cuales se pretende estimular la emergencia de actitudes positivas frente a la existencia de los denominados desechos sólidos.

Sin embargo, la realidad de la región se ha caracterizado por la presencia determinante de planes, sin una concreción efectiva en el aprendizaje y modelado de conductas ambientalistas, como consecuencia de la interacción limitada entre el contenido en esta área y la actividad del estudiante. Según Yélamos (ob.cit.) "El problema radica en la significación que el ambiente presenta para el alumno" (pág. 31). Es decir, la cultura ambientalista depende de una conexión entre los contenidos de Educación Ambiental y la realidad del estudiante, para que la misma representar un 
verdadero interés de aprendizaje.

Esta es una situación perfectamente observable en Venezuela, donde la cultura ambientalista y los contenidos de esta área de aprendizaje, no poseen un peso específico determinado por la calidad de las estrategias aplicadas en este sentido, según se desprende del trabajo de Montero (2011) quien señala: "No se le otorga a la educación ambiental, la importancia que posee, por cuanto se prefiere hacer énfasis en conocimientos prácticos como el matemático o el lenguaje" (pág. 9). Para el autor, la situación deriva de la concepción especifica en relación con las áreas de matemática y lectoescritura, hacia donde se dirigen básicamente los esfuerzos formativos.

Asimismo, en el interior del país, la situación es similar, definida por la importancia relativa del tema ambiental, el cual se considera como secundario frente a otras áreas de aprendizaje en mayores rangos de aplicación estratégica. Actividades como reutilización de desechos sólidos, son definidas como secundarios y normal o nominalmente adscritos a espacios como conexos a áreas relacionadas con geografía o salud. Es decir, no se les otorga un campo o consideración particulares.

Casos como el de la Escuela Básica Estadal Concentrada "Chaparral" determinan parte de la concepción señalada. En dicha institución, las actividades relacionadas con el manejo de variables ambientales, se definen en relación con otras áreas de aprendizaje y las mismas, no guardan una referencia efectiva con la gestión de un cambio conductual con respecto al ambiente y su protección. Actividades como el reciclaje, se limitan a acciones puntuales, definidas por la necesidad de uso de materiales de desecho en acciones aisladas.

Es importante señalar que las actividades estratégicas planteadas para el desarrollo de una cultura ambientalista, se limitan a exposiciones, carteleras o elaboraciones a partir de materiales reciclados, pero sin una valoración teórica de su importancia o actividades de refuerzo conductual, que motiven al 
estudiante para presentar un cambio de actitud frente a las variables ambientales que ocasionan problemas en su entorno vital. Esto se debe, según la observación inicial realizada a diversos factores entre los que se encuentran en primer término, la importancia relativa que identifica al área de Educación Ambiental en la institución.

Por otra parte, la reutilización de residuos sólidos como actividad fundamental del proceso de estímulo a una cultura ambientalista, es tratada como una experiencia limitada a la construcción de alternativas poco creativas. De hecho, se presentan estrategias en las que el mismo se relaciona con la elaboración de un elemento puntual, dirigido más a las artes plásticas y el trabajo manual que a la acción ambiental profunda que identifica el modelo de Educación Ambientalista. Se hace referencia tangencial a los valores de la protección del medio ambiente, pero no con la profundidad que el aprendizaje significativo implica.

\subsection{Objetivo de la investigación}

Proponer un plan estratégico para la reutilización de los residuos sólidos en la promoción de la cultura ambientalista en los estudiantes del sexto grado de la Escuela Básica Estadal "Chaparral”, durante el año escolar 2015-2016.

\subsection{Antecedentes.}

Ponte (2012) realizo un trabajo, identificado como Manejo integrado de residuos sólidos, Programa de reciclaje, para el Instituto Pedagógico de Caracas. En el mismo, se estableció como objetivo presentar un proyecto de reciclaje de papel que se realiza en el Instituto Pedagógico de Caracas (IPC) contemplado dentro de un plan de manejo integrado de residuos sólidos. El programa se inició en el año 2009 y cuenta con la participación de toda la comunidad y en especial con los estudiantes del curso de extensión acreditable de manejo de residuos sólidos. El programa parte del territorio e 
incide sobre el territorio constituyendo un modelo de gestión local. Los datos cuantitativos revelan los cambios positivos hacia la reutilización de residuos sólidos de papel por el incremento en la participación y en el papel recolectado. En la espiral de problemas - soluciones se plantea continuar con el reciclaje de latas, plástico y vidrio.

Hernández y Márquez (2013), presentaron el trabajo de investigación identificado como Estrategias pedagógicas para el estímulo al ambientalismo, como proceso educativo, el objetivo del estudio fue proponer estrategias pedagógicas para el estímulo a la Educación Ambiental, en los estudiantes y docentes del quinto año sección "A", del Liceo Bolivariano "Elías Araque Müller", parroquia Santa Bárbara, municipio Ezequiel Zamora del estado Barinas. En función de los objetivos planteados, se desarrolló una metodología de naturaleza cuantitativa, en un tipo descriptivo, asimismo, se presenta la modalidad del denominado proyecto factible, a través de un diseño de campo. En los resultados se pudo notar que el nivel estratégico utilizado por los docentes es limitado y la comprensión del tema, es decir, la motivación a la cultura ambientalista, por parte de los educandos, señala una necesidad estratégica, que se accede a través de la propuesta presentada como fase final de la investigación.

\section{Teoría y Conceptos.}

\subsection{Residuos Sólidos}

En la actualidad, la cultura del ser humano ha convertido a las personas en instrumentos de contaminación, a medida que mejora la situación económica aumenta el poder adquisitivo y con esto se incrementa la producción de residuos sólidos, con respecto al tema Chacin (2009) expresa:

Es todo material considerado como desecho y que se necesita eliminar. La basura es un producto de las actividades humanas al cual se les considera de valor igual a cero por el desecho. No necesariamente debe ser odorífica repugnante e 
indesechable eso depende del origen y composición de esta normalmente se le coloca en lugares predestinados para la recolección. Para ser canalizados a tiraderos o vertederos rellenos sanitarios y otros lugares. Actualmente se usa ese término para denominar aquella fracción de residuos que no son aprovechables y que por lo tanto debería ser tratada y dispuesta para evitar problemas sanitarios o ambientales (pág. 2).

Precedente a la opinión anterior, es relevante realizar el trabajo de sensibilización basado sobre la creación de una nueva cultura ambiental, lo se traduce en la formación de ciudadanos con conciencia ecológica capaces de reconocer sus problemas ambientales locales; solo a través del cambio de pensamiento de la sociedad se podrá disminuir la producción de residuos contaminantes.

\subsection{Reutilización de los residuos sólidos}

La actividad de reutilización de desechos sólidos supone además de un ahorro de recursos, una reducción en el uso de energía, en los procesos de producción y en definitiva una racionalización del uso de los recursos. Según Ponte (2012): "El reciclaje de los materiales de desecho implica: (a) recuperación de los materiales del flujo de desechos, (b) el procesamiento intermedio, por ejemplo, la selección y la compactación, (c) el transporte y (d) el procesamiento final, para dar origen a nuevos productos" (pág. 14). Los aspectos principales a considerar en el reciclaje de materiales incluyen: la identificación de los materiales que se van desviar de la corriente de desechos, las posibilidades de reutilización y reciclaje, así como las especificaciones de los compradores de materiales recuperados

Con respecto a la construcción de las bases teóricas de la investigación es imprescindible abordar el tema de la cultura ambiental, según Cruz (2008) es "entendida como aquella postura ante la vida que nos permite cuidar y 
preservar nuestro medio ambiente, es un asunto de interés para todo el mundo". (pág. 1). De acuerdo con esta opinión es fundamental promover la formación de una cultura orientada a la formación en valores ambientales. Cuando se habla de cultura a forma de vida caracterizada por lo que se hace y como se piensa; lo cual representa un conjunto de saberes que transmite de una generación a otra, dándole su respectiva particularidad.

\subsection{Estrategias didácticas en la Educación Ambiental}

Con respecto al tema Cano (2009), afirma que una estrategia didáctica es "Un conjunto de actividades que por su estructura orgánica incrementa la probabilidad de obtener un determinado logro" (pág. 42). Por tanto, promueve la expresividad y la creatividad y requiere de un espacio pedagógico, en el cual se interrelacionen la realidad social, los sentimientos patrios y las emociones individuales. Es decir, se consideran las dimensiones cognitiva y afectiva tomando en cuenta el entorno escolar. Es necesario enfocar la elaboración, planificación y ejecución de las estrategias didácticas de la investigación bajo el enfoque de la pedagogía, al ser considerada como el estudio sistemático y riguroso que se hace sobre la formación de un individuo.

\section{Metodología.}

\subsection{Naturaleza, tipo y diseño de la investigación}

La naturaleza del presente trabajo se inscribe en la cuantitativa, descrita por Miguel (1997) citado por Pérez (2013) según el siguiente concepto: "Es aquella que se dirige a recoger información objetivamente mensurable" (pág. 63). Esta consideración implica que los datos recolectados, deben ser efectivamente medibles a través de las técnicas dispuestas por la investigadora para tal finalidad, definiendo para ello el carácter objetivo de tal acción. Con relación al tipo, se caracteriza por ser un proyecto factible como complemento del tipo de investigación, definido por la Universidad Pedagógica 
Experimental Libertador, (2006) como:

La investigación, elaboración y desarrollo de un modelo operativo viable para solucionar problemas, requerimientos necesidades de organizaciones o grupos sociales que pueden referirse a la formulación de políticas, programas, tecnologías, métodos, o procesos. El proyecto debe tener el apoyo de una investigación de tipo documental, y de campo, o un diseño que incluya ambas modalidades (pág. 16).

En efecto, se hace referencia a un modelo de operaciones que pueden utilizarse en la solución de la situación problemática observada, el estudio presenta un diseño de campo como apoyo en el funcionamiento del proyecto el cual es descrito por la Universidad Pedagógica Experimental Libertador (2006) como:

El análisis sistemático de problemas en la realidad, con el propósito bien sea de describirlos, interpretarlos, entender su naturaleza y factores constituyentes, explicar sus causas y efectos, o predecir su ocurrencia, haciendo uso de métodos característicos de cualquiera de los paradigmas o enfoques de investigación conocidos o en desarrollo. (pág. 11).

De esta forma, se cual determina el procedimiento a ser aplicado en las fases posteriores del trabajo ello debido a que el fenómeno, será estudiado directamente en el ámbito y espacio humano en el cual se produce, sin trasladarlo o modificarlo en sus elementos constitutivos

\subsection{Población y Muestra}

Se estima necesario obtener las informaciones correspondientes de las personas involucradas en la situación que se desea comprender. A este grupo de individuos se le identifica como población o universo. El mismo es descrito por Hurtado y Toro (2008), como: "El total de los individuos o elementos a quienes se refiere la investigación" (pág. 31). En este caso, la población se corresponde con la totalidad de los docentes. Dicha cifra alcanza el total de 
cinco (05) docentes que laboran en la Escuela Básica Estadal "Chaparral", durante el año escolar 2015 - 2016. Una vez seleccionada la población dentro de la investigación se procedió a establecer la muestra. Según Arias (2006) "la muestra es un subconjunto representativo y finito que se extrae de la población accesible" (pág. 83). Para ello se tomó el 100\% de la población docente por considerarse una población pequeña.

\subsection{Técnicas e Instrumento de Recolección de datos}

En este caso, se ha seleccionado la encuesta la cual es descrita por Arias (2006), como: "Una técnica que pretende obtener información que suministra un grupo o muestra de sujetos acerca de si mismos, o en relación con un tema particular" (pág. 72). De acuerdo con esto, la persona seleccionada como parte de la muestra, facilita los datos de manera individual y por ende, determina su opinión en el procedimiento planteado. Por otra parte, en el caso del instrumento, se ha seleccionado el cuestionario, como el más idóneo para ser aplicado en este tipo de muestras. Según Arias (2006) el mismo: "Se realiza de forma escrita mediante un instrumento o formato en papel contentivo de una serie de preguntas. Se le denomina cuestionario auto administrado porque debe ser llenado por el encuestado, sin intervención del encuestador" (pág. 74). De esta manera, se facilitan los procedimientos de inferencia y conclusiones del estudio, a través de un mecanismo sencillo, susceptible de ser interpretado mediante el uso de la estadística descriptiva. Ahora bien, el diseño del cuestionario a ser presentado, se realizará en un escalonamiento tipo Likert con respuesta cerrada, en tres opciones de respuestas posibles Siempre (S) A Veces (A V) o Nunca (N), para cada una de las ocho (8) interrogantes planteadas, e identificadas por los indicadores de la operacionalización de variables anteriormente presentada. 


\subsection{Validez y Confiabilidad}

Para la validez se utilizó el juicio de expertos, especialista en metodología y educación ambiental y, para la confiabilidad se utilizó la prueba estadística Alpha de Cronbach, generando como resultado 0.83 , definido por Hernández, Fernández, y Baptista (2010), como "el grado en que su aplicación repetida al mismo sujeto u objeto producen los mismos resultados" (pág. 212). Para el análisis de los resultados. Los distintos datos se presentaron de manera tal que pueden ser interpretados de manera gráfica a través de diseños específicos de identificación de los datos por cada indicador propuesto en la operacionalización de variables, así como su correspondiente interpretación. Para cumplir con esta fase se utilizarán herramientas informáticas, como los programas Word y Excel en la construcción de los gráficos circulares y análisis de los ítems.

\section{Resultados.}

Posteriormente de la aplicación de las encuestas se llevó a cabo el análisis pertinente de la información aportada por los docentes la cual es de gran incidencia para la elaboración del plan estratégico, los datos recolectados ofrecen el diagnóstico preciso para la consecución del trabajo investigativo.

Cuadro $\mathbf{N}^{\circ}$ 1. Operativos de limpieza junto a los estudiantes para recolectar residuos sólidos.

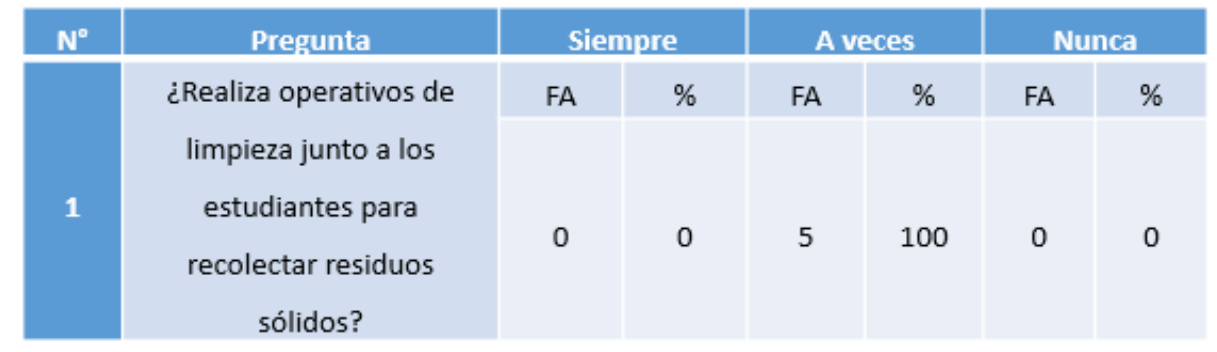

Fuente: Aguilera (2016) 
Dentro de los hallazgos se evidenció que la totalidad de los docentes a veces realizan operativos de limpieza, dejando abierta la oportunidad de realizar esta actividad con mayor continuidad y con un carácter pedagógico que contribuya con la formación valores ambientales en los estudiantes.

Cuadro $\mathbf{N}^{\circ}$ 2. Orientación a los alumnos en la clasificación de los residuos sólidos que se producen en la institución.

\begin{tabular}{|l|l|l|l|l|l|l|l|}
\hline \multicolumn{1}{|c|}{ Pregunta } & \multicolumn{2}{|c|}{ Siempre } & \multicolumn{2}{|c|}{ A veces } & \multicolumn{2}{|c|}{ Nunca } \\
\hline
\end{tabular}

Fuente: Aguilera (2016)

Con respecto a la orientación que los docentes brindan a los estudiantes sobre la clasificación de los residuos sólidos, el 20 \% de la muestra afirmó que siempre orienta a sus alumnos en la buena disposición de los residuos en la institución; mientras que el $80 \%$ respondió que a veces. Esta información es muy importante porque permite visualizar que existe una buena base para el proceso de formación sobre la reutilización de materiales

Cuadro $\mathbf{N}^{\circ}$ 3. Promoción de actividades de reutilización de residuos sólidos.

\begin{tabular}{|c|c|c|c|c|c|c|c|}
\hline $\mathbf{N}^{\circ}$ & Pregunta & \multicolumn{2}{|c|}{ Siempre } & \multicolumn{2}{|c|}{ A veces } & \multicolumn{2}{|c|}{ Nunca } \\
\hline & ¿Promueve actividades & FA & $\%$ & FA & $\%$ & FA & $\%$ \\
\hline 3 & $\begin{array}{l}\text { de reutilización de los } \\
\text { residuos sólidos? }\end{array}$ & 3 & 60 & 2 & 40 & 0 & 0 \\
\hline
\end{tabular}

Fuente: Aguilera (2016)

El $60 \%$ de los encuestados respondió que siempre realiza actividades para promover la reutilización de los residuos sólidos, mientras que el $40 \%$ 
restante opinó que a veces se ejecutan este tipo de actividades. De acuerdo con esta información es deducible la factibilidad del desarrollo de un plan para el aprovechamiento de los materiales reusables, pariendo de la premisa de que los estudiantes están familiarizados con la temática ambiental.

Cuadro $\mathbf{N}^{\circ}$ 4. Promoción de actividades para fortalecer los valores ambientales.

\begin{tabular}{|c|c|c|c|c|c|c|c|}
\hline $\mathbf{N}^{\circ}$ & Pregunta & \multicolumn{2}{|c|}{ Siempre } & \multicolumn{2}{|c|}{ A veces } & \multicolumn{2}{|c|}{ Nunca } \\
\hline & ¿Promociona actividades & FA & $\%$ & FA & $\%$ & FA & $\%$ \\
\hline 4 & $\begin{array}{c}\text { para fortalecer los valores } \\
\text { ambientales? }\end{array}$ & 5 & 100 & 0 & 0 & 0 & 0 \\
\hline
\end{tabular}

Fuente: Aguilera (2016)

El $100 \%$ de los encuestados respondieron que siempre realiza actividades para fortalecer los valores ambientales. De acuerdo con estos datos, la formación ambiental de los estudiantes está integrada al que hacer educativo, lo que permite mayor viabilidad al desarrollo de los proyectos relacionados con la conservación del ambiente.

Cuadro $\mathbf{N}^{\circ}$ 5. Establecimiento de normas junto a los alumnos para la conservar el ambiente.

\begin{tabular}{|c|c|c|c|c|c|c|c|}
\hline$N^{\circ}$ & \multicolumn{2}{|c|}{ Pregunta } & \multicolumn{2}{|c|}{ A veces } & \multicolumn{2}{|c|}{ Nunca } \\
\hline
\end{tabular}

Fuente: Aguilera (2016)

El $100 \%$ de los encuestados respondieron que siempre establecen normas junto a los alumnos para la conservación del ambiente. De acuerdo con estas aseveraciones, es importante señalar que el establecimiento de 
normas es esencial para la organización y la ejecución de proyectos factibles orientados a la formación de la ética ambiental.

Cuadro № 6. Promoción de cambios de conducta hacia la formación de la cultura ambiental.

\begin{tabular}{|c|c|c|c|c|c|c|c|}
\hline $\mathbf{N}^{\circ}$ & Pregunta & \multicolumn{2}{|c|}{ Siempre } & \multicolumn{2}{|c|}{ A veces } & \multicolumn{2}{|c|}{ Nunca } \\
\hline & ¿Promueve cambios de & FA & $\%$ & FA & $\%$ & FA & $\%$ \\
\hline 6 & $\begin{array}{c}\text { conducta hacia la } \\
\text { formación de la cultura } \\
\text { ambiental? }\end{array}$ & 0 & 0 & 5 & 100 & 0 & 0 \\
\hline
\end{tabular}

Fuente: Aguilera (2016)

El $100 \%$ de los encuestados respondieron que a veces promocionan cambios de conducta hacia la formación de la cultura ambiental. De acuerdo con la información ofrecida por los educadores, las actividades que ocasionan cambios conductuales requieren de mayor rigurosidad y frecuencia, lo cual amerita una planificación estratégica que permita realizar seguimiento en el avance de la formación de nuevos valores.

Cuadro $\mathbf{N}^{\circ}$ 7. Desarrollo de contenidos orientados a la promoción de la cultura ambiental.

\begin{tabular}{|c|c|c|c|c|c|c|c|}
\hline $\mathbf{N}^{\circ}$ & Pregunta & \multicolumn{2}{|c|}{ Siempre } & \multicolumn{2}{|c|}{ A veces } & \multicolumn{2}{|c|}{ Nunca } \\
\hline & ¿Desarrolla contenidos & FA & $\%$ & FA & $\%$ & FA & $\%$ \\
\hline 7 & $\begin{array}{l}\text { orientados a la } \\
\text { promoción de la cultura } \\
\text { ambiental? }\end{array}$ & 5 & 100 & 0 & 0 & 0 & 0 \\
\hline
\end{tabular}

Fuente: Aguilera (2016)

El $100 \%$ de los docentes encuestados respondieron que siempre desarrollan contenidos orientados a la promoción de la cultura ambiental. En 
este sentido, los datos aportados, permiten diseñar un plan estratégico partiendo sobre la base de que los estudiantes poseen una visión clara sobre algunos términos básicos, útiles en la formación del ser humano crítico, sensible y capaz de dar respuestas a problemas ambientales comunes.

Cuadro № 8. Aplicación de estrategias didácticas parta la promoción de la cultura ambiental.

\begin{tabular}{|c|c|c|c|c|c|c|c|}
\hline $\mathbf{N}^{\circ}$ & Pregunta & \multicolumn{2}{|c|}{ Siempre } & \multicolumn{2}{|c|}{ A veces } & \multicolumn{2}{|c|}{ Nunca } \\
\hline & ¿Aplica estrategias & FA & $\%$ & FA & $\%$ & FA & $\%$ \\
\hline 8 & $\begin{array}{c}\text { didácticas para la } \\
\text { promoción de la cultura } \\
\text { ambiental? }\end{array}$ & 5 & 100 & 0 & 0 & 0 & 0 \\
\hline
\end{tabular}

Fuente: Aguilera (2016)

El $100 \%$ de los encuestados respondieron que siempre aplican de las estrategias didácticas para la promoción de la cultura ambiental. Los datos aportados por los docentes son realmente interesantes para el proyecto en ejecución, los educadores poseen suficientes conocimientos que pueden ser aprovechados en plan estratégico para promover la cultura ambiental en los estudiantes.

\section{Conclusiones.}

De acuerdo con los hallazgos obtenidos de la encuesta aplicada a los docentes. Se evidencia que existen las teorías pertinentes, el talento humano capacitado, los recursos materiales y la compilación de estrategias requeridas, lo cual hace factible el desarrollo del plan estratégico para la reutilización de los residuos sólidos en la promoción de la cultura ambientalista en los estudiantes del sexto grado de la Escuela Básica Estadal "Chaparral", durante el año escolar 2015-2016. 
Para la promoción de la cultura ambientalista en los estudiantes del sexto grado de la Escuela Básica Estadal "Chaparral", durante el año escolar 2015-2016, Se realizó una revisión de fuentes documentales y electrónicas, se consultaron algunas personas de la comunidad con habilidades para la reutilización de residuos sólidos. Después de organizar los contenidos los materiales y el talento humano se diseñó el Plan estratégico el cual contemplo estrategias relacionados con la sensibilización ambiental, la clasificación de los residuos domésticos y el aprovechamiento de materiales reusables.

Con respecto a la aplicación de las estrategias para la promoción de la cultura ambientalista. El plan se realizó de acuerdo con lo pautado, se llevó a cabo el saneamiento ambiental de la escuela y la comunidad; se orientó a los estudiantes en la clasificación de los residuos sólidos. Se profundizaron contenidos referentes a la concepción del ambiente y la relación con el hombre. También se abordó la sensibilización de los estudiantes con la elaboración de trípticos que luego fueron distribuidos en la comunidad. Posteriormente se realizaron objetos artesanales con plástico, así como también se aprovechó el papel. Para culminar el plan se elaboró el huerto escolar aprovechando los residuos orgánicos.

Dentro de la evaluación de la ejecución del plan estratégico. Se observó en todo momento la motivación de los asistentes, así mismo se evidenció el interés por seguir desarrollando actividades de reutilización, aprovechando los recursos de la comunidad. También es palpable la necesidad de profundizar los contenidos ambientales presentes en los programas de la Educación Bolivariana. Con relación a la formación de valores ambientales se evidenció el esfuerzo de los participantes por mejorar la cultura ambiental del hombre actual y el compromiso en seguir la formación ecológica.

Crear una cultura ambiental es una labor que requiere de tiempo de compromiso y lo más importante es la aplicación de estrategias sencillas, novedosas y atractivas a los estudiantes. En este sentido, el estudio realizado 
plantea actividades muy prácticas que pueden ser utilizadas en contextos similares, promocionando valores ambientales que potencian los contenidos inmersos en el programa de la Educación bolivariana.

\section{Referencias.}

Arias, F. (2006). El Proyecto de Investigación: Introducción a la Metodología. Científica. (5ta. ed.). Caracas: Episteme.

Chacin, K. (2009). Elaboración de objetos artesanales de materiales de desecho en la parroquia El Moralito. Recuperado de:

https://www.monografias.com/trabajos82/elaboracion-odjetosartesanales-materia-probecho/elaboracion-odjetos-artesanales-materiaprobecho.shtml

Cruz, R. (2008). La cultura ambiental desde la Educación Básica. [Documento en línea]. Recuperado de:

http://www.elsiglodetorreon.com.mx/noticia/344173.la-cultura-ambientaldesde-la-educacion-basic.html

Hernández, J. y Márquez, D. (2013). Estrategias pedagógicas para el estímulo al ambientalismo, como proceso educativo. Barinas: UNELLEZ.

Hernández, R. Fernández, C. y Baptista, P. (2013). Metodología de la investigación. 5ta Edición. México: McGraw-Hill.

Hurtado, I. y Toro, J. (2008). Paradigmas y métodos de investigación en tiempos de cambio. Maracay: CEC, S.A.

Pérez, G. (2013). Introducción a la Metodología de la Investigación. Venezuela: Red Escolar Nacional - RENa.

Ponte, C. (2012). Manejo integrado de residuos sólidos, Programa de reciclaje, para el Instituto Pedagógico de Caracas. Caracas: UPEL.

Universidad Pedagógica Experimental Libertador (2006). Manual de grado de especialización y maestría y tesis doctorales. Caracas: Autor.

Yélamos, M. (2013). Informe del Ministerio de Salud de la República Argentina. Buenos aires: Fondo de Difusión. 


\section{Isbely del Carmen Aguilera Osorio}

e-mail: Im19752005@gmail.com

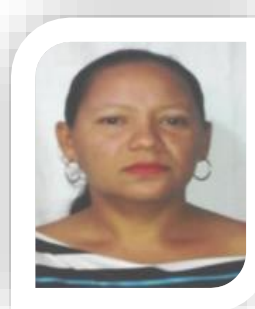

Nacida en Ciudad Bolivia. Estado Barinas. Cursa estudio de Maestría en Educación Ambiente y Desarrollo en la Universidad Pedagógica Experimental Libertador en Barinas Estado Barinas. Licenciada en Educación Integral en la Universidad Experimental de los Llanos Ezequiel Zamora. Se desempeña como docente de aula en la Escuela Básica estadal "Chaparral" del municipio Pedraza del Estado Barinas durante trece años. 